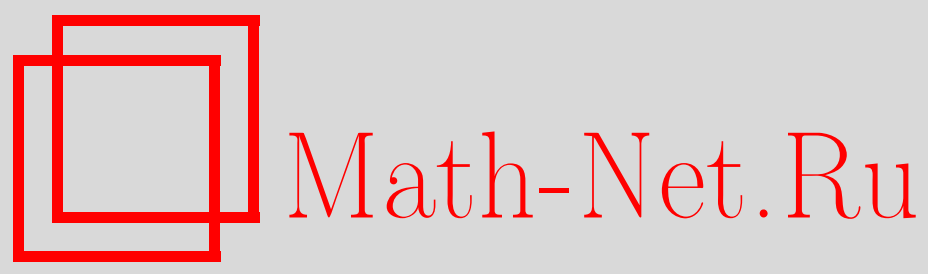

В. Д. Соловьев, Начальные сегменты эффективно-конечных порядков, Матем. заметки, 1997, том 61, выпуск 6, 950-953

DOI: https://doi.org/10.4213/mzm1581

Использование Общероссийского математического портала Math-Net.Ru подразумевает, что вы прочитали и согласны с пользовательским соглашением http://www.mathnet.ru/rus/agreement

Параметры загрузки:

IP: 54.81 .137 .203

26 апреля 2023 г., 05:25:00

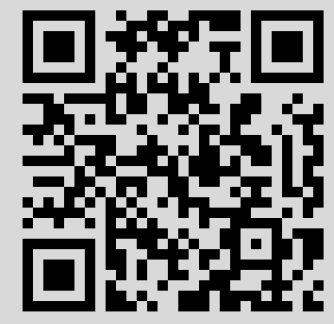




\section{НАЧАЛЬНЫЕ СЕГМЕНТЫ ЭФФЕКТИВНО-КОНЕЧНЫХ ПОРЯДКОВ}

\section{В. Д. Соловьёв}

Частичный порядок ' $\leqslant$ ' на ряде натуральных чисел $\mathbb{N}$, рассматриваемьй как бинарное отношение, является рекурсивно перечислимым, если множество пар $\langle x, y\rangle$ таких, что $x \leqslant y$ рекурсивно перечислимо. В ряде разделов теории алгоритмов встречаются рекурсивно перечислимые порядки, задаваемые более специальным образом.

Например, эффективная разметка натуральным рядом вершин бинарного дерева определяет рекурсивно перечислимый порядок, если положить $x \leqslant y$ тогда и только тогда, когда $y$ расположен на той же ветви, что и $x$, и $y$ ближе к корню, чем $x$. Характерной особенностью этого порядка является наличие двух функций $l(x)$ и $r(x)$, определяемых как левьй и правьй сьш вершины $x$. Эти две функции полностью определяют указанный вьше частичный порядок, причем $x \leqslant y \Longleftrightarrow x=y \vee \exists \tau(\tau-$ терм языка $\{l, r\}$ и $x=\tau(y))$.

Вводимые далее определения естественным образом обобщают частичные порядки, связанные с деревьями.

ОПРЕДЕЛЕНиЕ 1. Для данного набора $\mathscr{P}=\left\{f_{1}, \ldots, f_{n}\right\}$ частично рекурсивных функций частичный порядок ' $\leqslant \mathscr{P}$ ', задаваемьй условием $x \leqslant \mathscr{P} y \Longleftrightarrow x=y \vee \exists \tau(\tau-$ терм языка $\mathscr{P}$ и $x=\tau(y))$ назовем әффективно-конечным $\mathcal{M}$ определяемым набором $\mathscr{P}$.

ОПРЕДЕЛЕНИЕ 2. Начальным сегментом частичного порядка ' $\leqslant$ 'назьвается такое множество $S$, что $\forall x, y(x \leqslant y \& y \in S \Longleftrightarrow x \in S)$.

Легко видеть, что начальным сегментом эффективно-конечных порядков являются регрессивные множества и сплинтеры (определения этих понятий можно найти в [1]).

В первом случае эффективно-конечный порядок будет иметь минимальный элемент, а во втором - максимальньй.

Начальные сегменты эффективно-конечных порядков существенньм образом используются в теории программных алгебр рекурсивных функций. Так в [2] показано, что множество $\{f \mid f$ есть 1-местная общерекурсивная функция и $f(A) \subseteq A\} \cup\{1$-местные общерекурсивные предикаты $\}$ является максимальной подалгеброй тогда и только тогда, когда $A$ является начальньп сегментом некоторого эффективно-конечного порядка, определяемого набором общерекурсивных функций и обладающего наибольшим элементом.

Для алгебры частично рекурсивных функций имеет место аналогичньй результат с той разницей, что эффективно-конечньй порядок определяется набором частично рекурсивных функций.

Доказываемые в данной работе утверждения устанавливают существенную разницу в свойствах поря дков, определяемых наборами обще- и частично рекурсивных функций.

ПРЕДЛОЖЕНИЕ 1. Если $A$ - начальный сегмент әффективно-конечного порядка с наибольиим әлементом, определяемого набором общерекурсивных функиий, то $\bar{A}$ не гипериммунно.

Работа выполнена при финансовой поддержке Российского фонда фундаментальных исследований, грант № 93-011-16004. 
ДоКАЗАТЕЛЬСТво. Пусть набор $\mathscr{L}=\left\{f_{1}, \ldots, f_{n}\right\}$ определяет частичный порядок ' $\leqslant \mathscr{L}$ ' и $x_{0}$ - его наибольший элемент. Тогда либо $\bar{A}$ конечно, либо каждое из множеств

$$
E_{1}=\left\{x_{0}\right\}, \quad E_{2}=\bigcup_{i=1}^{n}\left\{f_{i}\left(x_{0}\right)\right\}, \quad E_{3}=\bigcup_{i=1}^{n}\left\{f_{i}\left(E_{2}\right)\right\}, \quad \ldots
$$

пересекается с $\bar{A}$ и, более того, каждое из множеств

$$
E_{1}^{\prime}=E_{1}, \quad E_{2}^{\prime}=E_{2}-E_{1}, \quad E_{3}^{\prime}=E_{3}-\left(E_{2} \cup E_{1}\right), \quad \ldots
$$

тоже пересекается с $\bar{A}$. Это следует из того, что $A$ - начальньй сегмент порядка ' $\leqslant \mathscr{L}$ ', поэтому если для какого-нибудь $E_{i}$ вьполнено $E_{i} \subseteq A$, то и $E_{i+1} \subseteq A$.

Система конечных множеств $\left\{E_{i}^{\prime}\right\}_{i=1}^{\infty}$ строится эффективньг образом, различные множества этой системы попарно не пересекаются и поэтому $\bar{A}$ не гипериммунно. Предложение 1 доказано.

ПРЕДЛОЖЕНИЕ 2. Существует рекурсивно перечислимое множсество с гипериммунным дополнением, являющееся начальным сегментом әффективно-конечного порядка, с наибольиим әлементом, определяемого набором частично рекурсивных функций.

ДокАЗАТЕЛЬСтво. Построение искомого множества $A$ будет вестись пошагово методом приоритета [1]. Параллельно с ним будут строиться две частично рекурсивные функции $f$ и $g$, которые и определяют эффективно-конечный порядок.

Обозначим через $A^{(n)}, f^{(n)}, g^{(n)}$ части множества $A$, функций $f$ и $g$ соответственно, построенные за $n$ шагов. Назовем число вакантным на шаге $n$, если оно не входит в область значений функций $f^{(n-1)}, g^{(n-1)}$. Построение будет вестись таким образом, что на каждом шаге невакантные числа образуют начальньй отрезок $N$.

Составим список всех частично рекурсивных функций: $\varphi_{0}, \varphi_{1}, \ldots$ - и будем вычислять параллельно (разумеется, это можно моделировать последовательным алгоритмом) значения всех частично рекурсивньх функций во всех точках, как это принято в теории алгоритмов [1]. В ходе построения некоторые функции из этого списка будут вычеркиваться.

В конструкции используются маркеры. В начальньй момент около всех чисел устанавливаются маркеры: около числа $i$ - маркер $i$. В ходе построения маркеры будут сдвигаться, причем так, что если число теряет маркер, то оно уже не может приобрести его в дальнейшем. Обозначим число, около которого стоит маркер $i]$ после $n$ шагов, через $a_{i}^{(n)}$.

Сдвиги осуществляются таким образом, что $\forall n \forall i \exists j \geqslant i\left(a_{i}^{(n+1)}=a_{j}^{(n)}\right)$. Множество $A^{(n)}$ после шага $n$ будут составлять числа, оставшиеся без маркеров.

Шаг 0. Пусть $A^{(0)}=\varnothing, f^{(0)}(0)=1$. Переходим к следуюшему шагу.

Шаг $n$. Для тех частично рекурсивных функций, которые еще не вычеркнуты из списка $\varphi_{0}, \varphi_{1}, \ldots$ и имеют номера $\leqslant n$, сделаем по $n$ шагов в вычислениях их значений в точках $0, \ldots, n$.

Пусть число $i$ удовлетворяет следующему свойству:

$(*) i$ - наименьшее число такое, что значения $\varphi_{i}^{(n)}(0), \ldots, \varphi_{i}^{(n)}\left(k_{i}\right)$ для некоторого $k_{i} \leqslant n$ все вычислились, $\mathscr{D}_{\varphi_{i}^{(n)}(0)}, \ldots, \mathscr{D}_{\varphi_{i}^{(n)}\left(k_{i}\right)}$ попарно не пересекаются, все 
имеют непустое пересечение с $A^{(n)}$ и для некоторого $j \mathscr{D}_{\varphi_{i}^{(n)}(j)}$ не содержит чисел $a_{0}^{(n-1)}, \ldots, a_{i}^{(n-1)}$.

Сдвигаем все маркеры $i^{\prime}, i^{\prime}>i$, таким образом, что маркер $i+1$ устанавливается около наименьшего вакантного числа, большего всех чисел из $\mathscr{D}_{\varphi_{i}^{(n)}(j)}$. Для всех $k>1$ маркер $i+k$ устанавливается около числа $a_{i+1}^{(n)}+k-1$. Остальные маркеры на этом шаге не сдвигаются.

Определяем на числе $a_{i}^{(n)}$ функцию $g$ как $a_{i+1}^{(n)}$, на числах от $a_{i}^{(n)}+1$ до $a_{i+1}^{(n)}-2-$ функцию $f(x)$ как $x+1$.

Вычеркиваем $\varphi_{i}$ из списка $\varphi_{0}, \varphi_{1}, \ldots$ частично рекурсивных функций.

Определяем функцию $f$ на числе $a_{i+1}^{(n)}$ равной $a_{i+1}^{(n)}+1$.

Если числа $i$ со свойством $(*)$ не найдется, то находим наименьшее вакантное число $x$, на котором функция $f$ еще не определена, и полагаем $f(x)=x+1$.

Построение завершено. Множество $A=\bigcup_{n} A^{(n)}$ есть множество чисел, оставшихся в итоге без маркеров; $f=\bigcup_{n} f^{(n)}$ и $g=\bigcup_{n} g^{(n)}$.

Докажем, что множество $A$ удовлетворяет требованиям предложения 2 в каждом из следующих случаев:

a) $\bar{A}$ бесконечно;

б) $\bar{A}$ гипериммунно;

в) $A$ рекурсивно перечислимо;

г) эффективно-конечньй порядок ' $\leqslant\{f, g\}$ ' обладает наибольшим элементом;

д) $A$ - начальньй сегмент порядка ' $\leqslant\{f, g\}$ '.

a) Покажем, что любой маркер сдвигается лишь конечное число раз. Действительно, маркер 0 не сдвигается вообще. Маркер 1 может сдвинуться лишь один раз при выполнении требования $(*)$ для функции $\varphi_{0}$, так как после этого $\varphi_{0}$ вычеркивается из списка частично рекурсивных функций. Аналогично, маркер $n$ может сдвигаться лишь при выполнении требования $(*)$ для функций $\varphi_{0}, \ldots, \varphi_{n}$, которые после этого вычеркиваются. Следовательно, $n$ сдвигается лишь конечное число раз.

Так как число, около которого стоит маркер в своей окончательной позиции, принадлежит множеству $\bar{A}$, отсюда следует, что $\bar{A}$ бесконечно.

б) Действительно, предположим противное. Если бы существовала такая общерекурсивная функция $\varphi_{t}$, что

$$
\forall x\left(\mathscr{D}_{\varphi_{t}(x)} \cap \bar{A} \neq \varnothing\right) \& \forall x, y\left(x \neq y \Longrightarrow \mathscr{D}_{\varphi_{t}(x)} \cap \mathscr{D}_{\varphi_{t}(y)}=\varnothing\right)
$$

то на некотором шаге $n>t$ вычислились бы $\varphi_{t}^{(n)}(0), \ldots, \varphi_{t}^{(n)}(t+1)$, и так как $\forall x, y$ $\left(x \neq y \Longrightarrow \mathscr{D}_{\varphi_{t}(x)} \cap \mathscr{D}_{\varphi_{t}(y)}=\varnothing\right)$, то найдется число $j$ такое, что $\mathscr{D}_{\varphi_{t}(j)}$ не содержит чисел с маркерами $0, \ldots, \mathbb{t}$ (т.е. $a_{0}^{(n-1)}, \ldots, a_{t}^{(n-1)}$ ). Тогда по конструкции все числа из $\mathscr{D}_{\varphi_{t}(j)}$ лишаются маркеров и, следовательно, включаются в множество $A$ в противоречие с допущением $\forall x\left(\mathscr{D}_{\varphi_{t}(x)} \cap \bar{A} \neq \varnothing\right)$.

в) Это следует из эффективности всего построения и того, что если число лишается маркера, т.е. попадает в $A$, то оно уже не может приобрести маркер вновь.

г) Наибольшим элементом является число 0. Действительно, из конструкции видно, что на любом шаге невакантные числа, т.е. являюшиеся значениями функций $f$ или $g$, образуют начальньй отрезок $N$. 
В пределе вакантные числа отсутствуют ввиду того, что $\bar{A}$ гипериммунно, оно не конечно и, следовательно, в конструкции имело место бесконечно много перемещений маркеров. Отсюда и из того, что функции $f$ и $g$ монотонно возрастающие, следует, что $\forall x \exists \tau(\tau-$ терм $\{f, g\}$ и $\tau(0)=x)$, т.е. число 0 - наибольший элемент порядка ' $\leqslant\{f, g\}$ '.

д) Действительно, включение $x \in A$ означает, что на некотором шаге $n$ был осушествлен сдвиг маркеров таким образом, что число $x$ лишилось маркера. В этом случае, если функция $g$ была определена на $x$, то она принимает в качестве значения число, также лишающееся на этом шаге маркера и потому принадлежащее $A$.

Функция $f$ на числе $x$ определяется на шаге $n$ или раньше тоже так, что $f(x)=x+1$, и она обладает маркером на шаге $n$. Значит, $f(x)$ принадлежит $A$. Предложение доказано.

НИИ математики и механики

Поступило

при Казанском государственном университете

\section{СПИСОК ЦИТИРОВАННОЙ ЛИТЕРАТУРЫ}

1. Роджерс Х. Теория рекурсивных функций и эффективная вычислимость. М.: Мир, 1972. 2. Соловьёв В. Д. // Изв. вузов. Матем. 1982. № 12. С. 51-56. 